\title{
Advanced models for AGN emission
}

\section{F. Spanier*}

Center for Space Research, North-West University, 2520 Potchefstroom, South Africa

E-mail: felixafspanier.de

\section{Weidinger}

Lehrstuhl Theoretische Physik IV, Ruhr-Universität Bochum, Germany

E-mail: mweidingeretp4.rub.de

\section{S. Richter}

Lehrstuhl für Astronomie, Universität Würzburg, Germany

E-mail: srichterdastro.uni-wuerzburg.de

Active Galactic Nuclei have been in the focus of gamma-ray telescopes for the past years. With the ever growing sample of AGN the need for physically motivated, self-consistent modeling is also growing.

The major questions to be answered by models are: What are the main constituents of AGN jets? What are the acceleration mechanisms? Are AGN possible accelerators for UHECR and a possible source of UHE neutrinos?

We will present new modeling approaches for AGN, which have a focus on the self-consistent approach. Two types of models have emerged from our work: A homogeneous model containing acceleration via Fermi mechanisms, leptonic and photo-hadronic radiation mechanisms and time variability. And a spatially extended model containing the same radiation processes, but spatially resolved shock structures and a non-isotropic particle distribution.

The results contain a possible discrimination criteria for hadronic and leptonic radiation models and timing effects of spatial dependence.

The 34th International Cosmic Ray Conference,

30 July- 6 August, 2015

The Hague, The Netherlands

\footnotetext{
* Speaker.
} 


\section{Introduction}

The observation of Active Galactic Nuclei (AGN) has seen a major breakthrough in the last decades thanks to the success of ground-based Cerenkov telescopes and satellite missions like Fermi. Very detailed multi-frequency campaigns of some sources (e.g. [1, 2, 3]) were giving a very precise picture of the emission of AGN. This picture nowadays no longer consists of static snapshots, but for well observed sources also very detailed light curves. This in turn allows for the derivation of more complete, physics driven models. While a single snapshot of the spectral energy distribution (SED) may still be described using simple steady-state models, observed variability requires time-dependent models, which should be self-consistent to base the model purely on a small set of physical input quantities.

One of the most important questions with regard to AGN in the past has always been, whether hadronic processes play any role in the emission of AGN. It seems at least as if distant sources of the FSRQ type are more likely to be hadron influenced than nearby HBL type AGN. Regardless of the presence of hadrons, leptonic processes will contribute to the radiation in any source. For the case of variable sources, the question how particles are accelerated and what the cause of variability is. The latter question cannot be answered yet by observations, but the blob like structures and the presence of shock waves suggests scenarios, in which an emitting region transverses zones of different magnetic field or density.

Variability, acceleration mechanism and type of emitting particles are interlinked with each other in many different ways. The acceleration mechanism determines the spectrum of emitting particles and the acceleration timescales for the different particles. Time-dependent models employing an explicit acceleration mechanism will, therefore, yield information on this mechanism and the particles involved. Sources with radiation of hadronic origin, have far more complicated light curves than purely leptonic sources.

A very important topic is nowadays also the ultra short time variability, which has been observed in at least two Blazars $[4,5]$. The observed times of the order of minutes lead, even for very high Doppler factors, to length scales much shorter than the regions assumed in the homogeneous Synchrotron Self Compton (SSC) paradigm. Under the assumption that the flare originates from the same region as the steady state emission, a spatially resolved modeling of this region, taking into account the light travel times, is necessary.

\section{Models}

We describe the details of the two models used for this work. The first one, modell, is a time dependent, two-zone, hybrid code [6]. The second one, model2, is a spatially resolved SSC code, able to trace the acceleration process back to the jets microphysics [7].

\subsection{Lepto-Hadronic models}

The hybrid model has a simple geometrical structure, which resembles a spherical blob travelling down the jet axis towards the observer with a Lorentz factor $\Gamma$. Upstream material is injected with $Q_{0, i}(\gamma)=Q_{0, i} \delta\left(\gamma-\gamma_{0, i}\right), i$ being the particle species. The blob has two zones nested into each other: A small acceleration zone with strong turbulence and a large radiation zone with low 
acceleration efficiency.

Particles undergo diffusive shock acceleration in the acceleration zone. The time scale for this process in the hard sphere limit is given by

$$
t_{\mathrm{acc}, i}=\left(\frac{v_{s}^{2}}{4 K_{\|, i}}+2 \frac{v_{A}^{2}}{9 K_{\|, i}}\right)^{-1} \propto m_{i}
$$

with $v_{s}$ and $v_{A}$ as the typical shock and Alfvén speeds. The timescale is given in terms of the gyration time, which then relates it to the particle's mass. The escape time scale is a constant related to the acceleration time scale $t_{\mathrm{esc}, i} \propto t_{\mathrm{acc}, i}$. This ensures power-law particle spectra, as expected from DSA [8]. The kinetic equations in the acceleration zone, as derived from the relativistic Vlasov equation, read as follows:

$$
\partial_{t} n_{i}=\partial_{\gamma}\left[\left(\beta_{s, i} \gamma^{2}-t_{a c c, i}^{-1} \gamma\right) \cdot n_{i}\right]+\partial_{\gamma}\left[\left[(a+2) t_{a c c, i}\right]^{-1} \gamma^{2} \partial_{\gamma} n_{i}\right]+Q_{0, i}-\frac{n_{i}}{t_{e s c, i}}
$$

with the synchrotron $\beta_{s, i} \propto B m_{i}^{-3}$ and $a \propto v_{s} / v_{A}$.

All particles escaping the acceleration zone enter the radiation zone where particle acceleration is negligible. Here, the particles are confined long enough to ensure efficient emission, hence all relevant processes have to be taken into account. Considering $p^{+}$confinement this requires relatively high $B$-fields (as compared to SSC [8]) of $\mathscr{O}(10 \mathrm{G})$ to guarantee enough gyrations.

Synchrotron photons and non-thermal protons produce pions through various channels. The produced pions will decay into stable $e^{ \pm}$(and $\gamma \mathrm{s}$ ). These leptons will have ultra-relativistic energies. Their synchrotron emission and the $\gamma_{\mathrm{s}}$ from $\pi^{0}$ decay will lead to pair-production. This will initiate an electromagnetic cascade until the radiation enters the optically thin regime. The radiation zone can be described by the following kinetic equations:

$$
\partial_{t} N_{e^{ \pm}}=\partial_{\gamma}\left[\left(\beta_{s, e} \gamma^{2}+P_{I C}\right) \cdot N_{e^{ \pm}}\right]-\frac{N_{e^{ \pm}}}{t_{r a d, e s c, e}}+Q_{p p}+Q_{p \gamma^{ \pm}}+b \frac{n_{e^{ \pm}}}{t_{e s c, e}}
$$

with $n_{e^{+}}=0$ (no primary positrons), and for the protons

$$
\partial_{t} N_{p^{+}}=\partial_{\gamma}\left[\left(\beta_{s, p^{+}} \gamma^{2}+P_{p \gamma}\right) \cdot N_{p^{+}}\right]+b \frac{n_{p^{+}}}{t_{e s c, p^{+}}}-\frac{N_{p^{+}}}{t_{e s c, r a d, p^{+}}} .
$$

$b$ is a constant geometric factor ensuring particle conservation. Analogous to the acceleration zone, we assume the escape timescale to be $t_{\mathrm{esc}, \mathrm{rad}, i} \propto m_{i}$.

The production rate of the stable particles resulting from pion production, $Q_{p \gamma^{ \pm}}\left(\gamma_{e^{ \pm}}\right) \propto N_{p^{+}}, N_{p h}$, is calculated using the $\Phi_{ \pm}$-parametrization of the full SOPHIA Monte Carlo calculations [9] carried out by [10]. Hence the synchrotron losses of the unstable intermediate $\mu^{ \pm}$are not accounted for. Even in relatively high magnetic fields this error remains small [6]. The pair-production rate $Q_{p p}(\gamma) \propto N_{\text {ph }}$ is calculated using the approximation Eq. (12) of [11] and the IC losses $P_{I C}\left(\gamma_{e^{ \pm}}\right) \propto$ $N_{\text {ph }}$ exploit the full Klein-Nishina cross section. To calculate the model SED the photon equation of the radiation zone Eq. 2.5 is beamed towards the observer, allowing for its redshift.

$$
\partial_{t} N_{\mathrm{ph}}=R_{s}+R_{c}+R_{\pi^{0}}-c\left(\alpha_{S S A}+\alpha_{p p}\right) N_{\gamma}-\frac{N_{p h}}{t_{p h, e s c}}
$$

The emissivities are $R_{c}(v) \propto N_{e^{ \pm}}, N_{\mathrm{ph}}, R_{\pi^{0}}(v) \propto N_{p^{+}}, N_{\mathrm{ph}}, R_{s}(v) \propto N_{e^{ \pm}}$for Compton scattering, neutral pion decay and synchrotron radiation respectively. The photon escape timescale is the light 
crossing time. For VHE photons the blob is optical thick due to $e^{ \pm}$-pair-production.

Making full use of the numerical implementation of the Eqs.2.2 to 2.5 a time-dependent treatment, including all non-linearities due to their couplings, is feasible. Blazar variability may hence be used to identify AGN accelerating protons to the highest energies revealing typical fingerprints (timescales as well as time lags) in their inter band lightcurves, see Sect. 3.

The treatment presented here has only a minimal set of additional parameter compared to SSC: The density ratio of injected electrons to protons and the energy of injected protons.

\subsection{Spatially-Extended Emission models}

The spatially resolved, time dependent model we present here, assumes a Fermi-I type acceleration process. This process is in fact a non-local process, since it needs a fluid shock and particle scattering centers far away from that shock. The here presented model therefore connects the acceleration process with the geometry of the simulation box, resulting from the spatial discretization. The necessary pitch angle scattering is included in a stochastically manner. This results in a ratio between acceleration and cooling time that depends on the distance to the shock.

The region around the shock is discretized in one dimension along the shock normal. In order to avoid a full discretization of the pitch angle $\mu=\cos (\theta)$, we use the fact that the pitch angle scattering will produce an isotropic particle distribution on short timescales. Furthermore no test particles are included and only the positions of the gyro centers are computed. These two points limit us to non-relativistic and non-oblique shocks. Consequently, the discretization is carried out along the magnetic field lines, parallel to the shock normal.

The resulting slices are indexed with $i$. One can then represent the shock by a jump in velocity of the ambient plasma. From the properties of the shock, its velocity $V_{S}$ and compression ratio $R$, the bulk velocity in each cell is calculated. It is not possible to include the pitch angle scattering in the full isotropic approximation. Therefore we divide the particle density into two bins, one for each half-space:

$$
n_{e l, i}^{+}(\gamma)=\int_{0}^{\pi / 2} n_{e l}\left(z_{i}, \gamma, \mu\right) \mathrm{d} \mu \quad n_{e l, i}^{-}(\gamma)=\int_{\pi / 2}^{\pi} n_{e l}\left(z_{i}, \gamma, \mu\right) \mathrm{d} \mu
$$

The introduction of a scattering rate between these two quantities (in the rest frame of the bulk plasma), together with the advection between cells, will lead to Fermi-I acceleration.

All other processes influencing the particle distribution are included in the time dependent kinetic equation, that is solved in each cell. It is derived from the Fokker-Planck equation by integrating over $\mu$ and separation of the resulting advection terms and is equivalent to Eq. 2.3. The evolution of the photon field is calculated via Eq. 2.5, except for the missing photohadronic terms and the pair-production-absorption. The total SED is then computed similar to the model of Blandford and Königl [12], taking into account light travel times and time dilation.

\section{Results}

\subsection{Flare scenarios for Markarian 501 using spatially resolved models}


The fits of the averaged low state of this source were produced with the model described in section 2.2 and are shown in Fig. 1. A good overall fit can be achieved, although the spectral index indicated by the UVOT and SMA data

\begin{tabular}{cccccc}
\hline \hline $\operatorname{sim}$ & $z(\mathrm{~cm})$ & $B(G)$ & $N_{\text {inj }}\left(\mathrm{s}^{-1}\right)$ & $\delta$ & $t_{\text {iso }}(\mathrm{s})$ \\
\hline $\operatorname{sim} 1$ & $4.5 \cdot 10^{15}$ & 0.044 & $8.9 \cdot 10^{43}$ & 33.5 & 5000 \\
$\operatorname{sim} 2$ & $3.8 \cdot 10^{15}$ & 0.06 & $8.25 \cdot 10^{43}$ & 32.1 & 125 \\
$\operatorname{sim} 3$ & $3.3 \cdot 10^{15}$ & 0.07 & $8.25 \cdot 10^{43}$ & 32.1 & 75
\end{tabular}

Table 1: Parameters obtained from the fits in Fig. 1. does not match the Fermi spectral index. The parameters summarized in table 1 for sim 1 differ from those presented by [1].

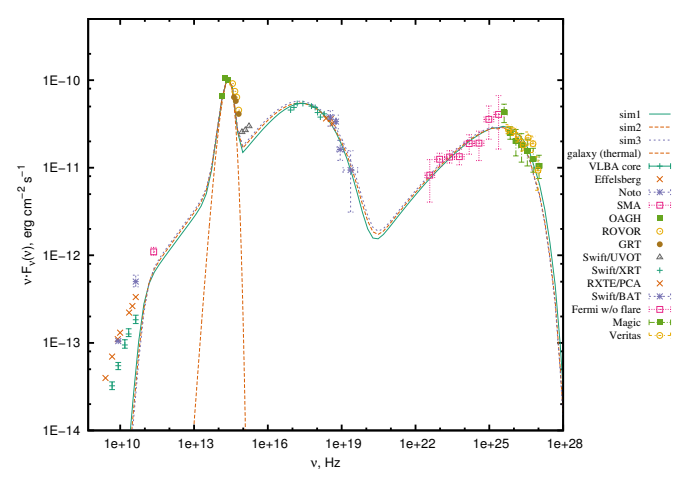

Figure 1: Fit of the data obtained during a multifrequency campaign [1].

The parameters result in a light crossing time of $t_{l c}=4545 \mathrm{~s}$. We want to stress that in the case $\left(t_{v a r}<t_{l c}\right)$ numerical studies of the variability are only possible with a spatially resolved model which preserves causality.

The parameter set $\operatorname{sim} 3$ is used to study different flare scenarios, here we focus on fast orphan flares. We have tested two different variations of the particle injection rate, as well as an external photon scenario, and a so called multiple shock scenario. The latter describes the possibility of reacceleration through additional shocks in the downstream. In [13] we have shown, that additional particle injection can not account for the observed variability timescale in the highest energies. The acceleration of particles from the injection energy up to the $\mathrm{TeV}$ scale on the observed timescale would require a much larger acceleration rate that is not compatible with the steady state SED.

An additional difficulty is the increase of inverse Compton cooling with higher particle densities. An increase by one order of magnitude, will lead to a flux reduction in the Whipple-band, unless the magnetic field is reduced simultaneously. This is on the one hand in contradiction with the equipartition assumption, on the other hand a lower magnetic field will further increase the cooling timescale.

In the multi-shock-scenario only particles with energies above the cooling break are effected, which is, close to the primary shock, only a narrow band. Consequently the system returns to the steady state very fast, after the secondary shock disappears. This disappearance is however artificially introduced. Starting from the steady state fits, the lightcurves in Fig. 3 were obtained by injection of an external photon distribution.

A synchrotron spectrum was chosen for the spectral shape. The two occurring parameters are the cut off frequency and the normalization in the form of the differential energy density $E_{p h, i n j, 0}$ at $v_{c}$. For both $\operatorname{sim} 2(\rightarrow$ IC flare 2$)$ and $\operatorname{sim} 3(\rightarrow$ IC flare 1$)$ fits were produced. The two parameters were set to $v_{c}=10^{16} \mathrm{~Hz}, E_{p h, i n j, 0}=6.6 \cdot 10^{-17} \mathrm{erg} \mathrm{s} \mathrm{cm}^{-3}$ for IC flare 1 and $v_{c}=10^{15} \mathrm{~Hz}$, $E_{p h, i n j, 0}=3.3 \cdot 10^{-16} \mathrm{erg} \mathrm{s} \mathrm{cm}^{-3}$ for IC flare 2, respectively.

In contrast to the previously discussed scenarios, here the falling flank of the lightcurve is inde- 


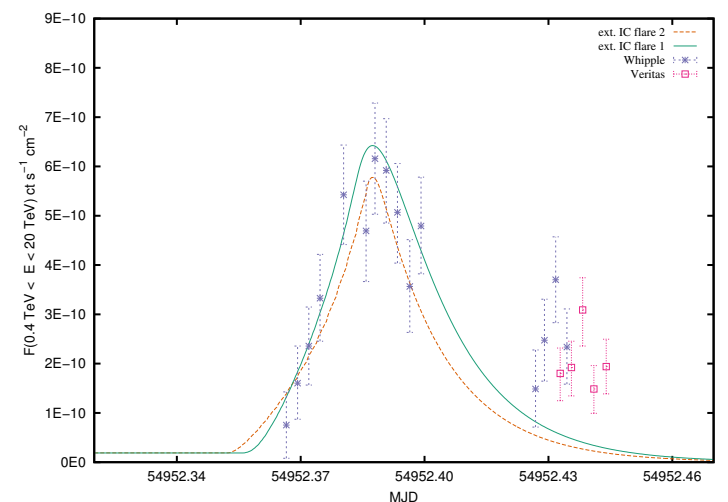

Figure 3: Fits of the lightcurve recorded by Whipple [4] employing the external inverse Compton scenario.

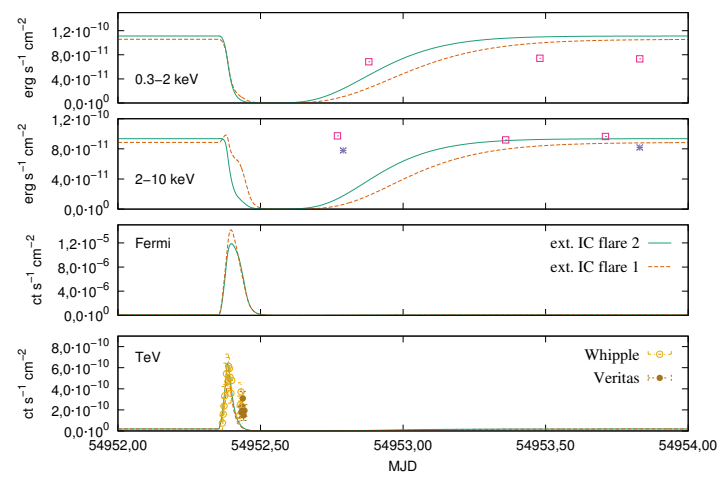

Figure 4: Simulated lightcurves in various energy ranges for the external inverse Compton flares.

pendent from the length of the injection and completely determined by inverse Compton cooling. In addition to the possible boosting this significantly relaxes the constraints on the source of the variability.

All presented flare scenarios produce variability in all relevant bands. An increase of the particle injection as well as the multiple shock scenario leads to a simultaneous increase in all bands, shown in Fig. 2. Only the hard X-ray flux stays approximately constant due to inverse Compton cooling in the injection case.

Here it is obvious, that even a magnetic field of almost $0.1 \mathrm{G}$ can neither explain the rapid rise and fall in the $\mathrm{TeV}$ range, nor the absence of an increased X-ray flux, approximately $6 \mathrm{~h}$ after the TeV-flare. The falling flank of the shock-scenario is sufficiently

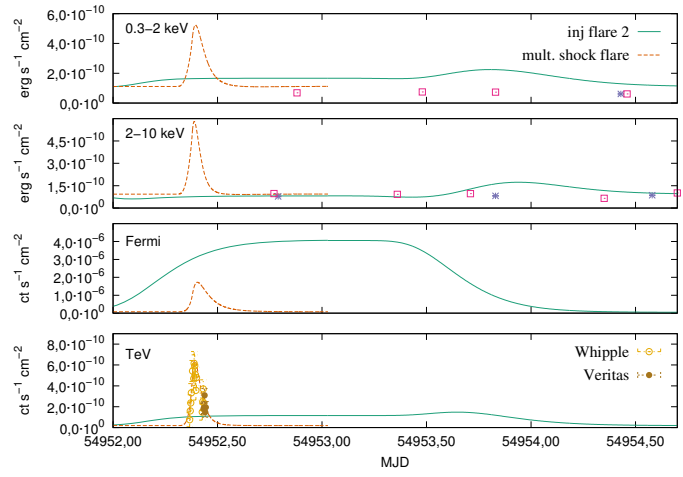

Figure 2: Simulated lightcurves in various energy ranges for particle injection and the multi shock scenario. steep.

The injection of a strong, additional photon field has an opposite effect on the electron synchrotron emission. As shown in Fig. 4 the X-ray emission is reduced as long as the external field in present. For the presented simulations this timespan is $t_{i n j} \approx 1.5 \cdot 10^{4} \mathrm{~s}$ in the observers frame. After stopping the injection the system returns to its steady state on the intrinsic timescale. It can be seen that $\operatorname{sim} 3$ is slightly faster, but can not explain the observed $\mathrm{X}$-ray flux.

\subsection{Hybrid emission model for 1 ES 1011+496}

The blazar 1 ES $1011+496$ is in a distance of $z=0.212$ and has originally been classified as high frequency peaked BL Lac object. The true nature of this blazar seems to be quite unclear compared to well studied objects like Mkn 501, PKS 1218+304 or 3C 279. The first and only multi 


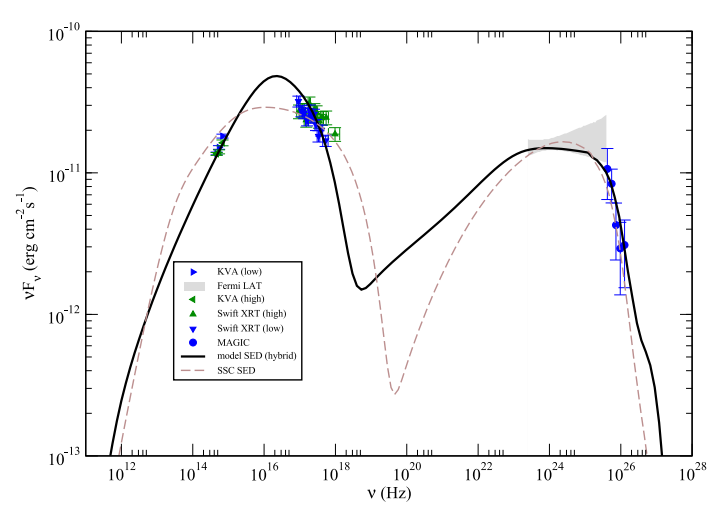

Figure 5: Simultaneous data from [14]. Blue symbols indicate the low emission state used in the modeling. The high state inferred from the slight variability is shown in green. The gray butterfly represents the first year catalog spectrum of Fermi LAT. The gray dashed curve displays the modeling attempt with a low magnetic field and no protons present in the jet (Table 2a), the black solid curve is due to the modeling with high $B$-field co-accelerated protons (Table 2b).

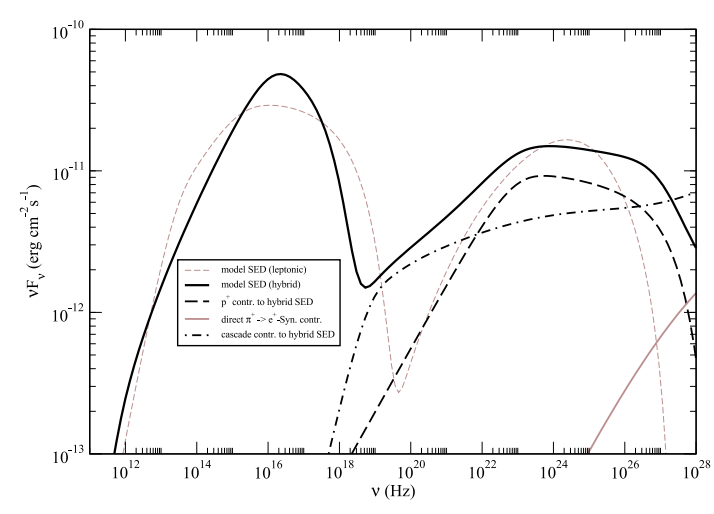

Figure 6: Intrinsic SEDs of 1 ES 1011+496 (Fig. 3.2) modeled with parameters from Table 2. The solid line shows the hybrid SED with the individual components: proton synchrotron emission (dashed black) and synchrotron emission by $e^{ \pm}$-pairs initiated by photohadronic interactions (dashed-dotted black) only observable due to pair-cascades. Direct contribution of stable end-products from photo-pion production (solid brown, $\pi^{+} \rightarrow e^{+}$) are negligible and affect the SED via $\gamma \gamma$-pair-production of the $n$th generation only.

wavelength observation, including MAGIC in the VHEs, Swift in X-rays and KVA in the optical band was in 2008 [14]. During this campaign the emission of 1 ES 1011+496 was more or less steady with slight spectral variability in the optical and X-rays having no counterpart in the $\gamma$-rays [14].

In Figure 3.2 the measured multi wavelength spectrum from [14] as well as two model SEDs are shown (here only EBL absorbed data points are used). The parameters of the models can be found in Table $2 \mathrm{a}$ and $2 \mathrm{~b}$ respectively. The rather hard spectrum in the optical combined with a photon index of $s=2.32$ in the Swift XRT band makes it difficult to explain the SED of 1 ES 1011+496 in terms of a self-consistent SSC-Ansatz. The best parameters in the Self-Compton limit, i.e. a low magnetic field unable to confine non-thermal protons within the considered region, are shown in Table 2a. A spectral index of 2.2 for the electrons with a magnetic field of $0.18 \mathrm{G}$ can neither reproduce the narrow synchrotron peak nor the spectral properties when computed selfconsistently. It ia possible to find a purely leptonic model SED for 1 ES 1011+496, see e.g. [14] itself, but in fact the underlying electron spectra are not the result of a self-consistent model. A physical explanation would have to be given a posteriori. Especially the cooling breaks produced by a self-consistent model pose a special problem: They fail to reproduce the synchrotron peak at low $B$-fields, automatically yielding a relatively high magnetic field being present within the jet. In such a case protons can be confined effectively and would add to the emission, leading to a hybrid spectrum of this particular blazar, see Fig. 3.2. The parameters used can be found in Table 2b. With the parameters shown in Table $2 b$ the measured MWL spectrum of 1 ES 1011+469 is explained well. The injected luminosity is $L_{p}=3.0 \cdot 10^{42} \mathrm{erg} \mathrm{s}^{-1}$ in hadrons, assuming a vanishing angle 


\begin{tabular}{lcccccccccc}
\hline \hline Model & $Q_{0, p^{+}}\left(\mathrm{cm}^{-3}\right)$ & $\gamma_{0, p^{+}}$ & $Q_{0, e^{-}}\left(\mathrm{cm}^{-3}\right)$ & $\gamma_{0, e^{-}}$ & $B(\mathrm{G})$ & $t_{a c c, e}(\mathrm{~s})$ & $R_{\text {blob }}(\mathrm{cm})$ & $t_{a c c} / t_{\text {esc }}$ & $a$ & $\delta$ \\
\hline a) & 0 & - & $7.50 \cdot 10^{4}$ & 868 & 0.18 & $3.5 \cdot 10^{4}$ & $8.00 \cdot 10^{15}$ & 1.2 & 1000 & 44 \\
b) & $1.55 \cdot 10^{8}$ & 600 & $3.78 \cdot 10^{7}$ & 3400 & 8.0 & $3.7 \cdot 10^{2}$ & $1.75 \cdot 10^{15}$ & 1.3 & 20 & 36 \\
\hline
\end{tabular}

Table 2: Parameters found in the modeling process to the multi wavelength data of 1 ES 1011+496 a) using low magnetic fields, b) high magnetic fields thus confined highly relativistic protons.

to the line of sight. This is significantly below the Eddington limit for the black hole assumed to be present in 1 ES 1011+496. To resolve the second peak in the spectrum it is much more convenient to take a look at the intrinsic SED unaffected by the EBL, shown in Fig. 6. The relevant contributions to the second peak of 1 ES 1011+496 are proton synchrotron photons of the highly relativistic primary $p^{+}$with Lorentz-factors up to $\gamma_{p} \approx 10^{10}$ and cascade radiation. As one can infer from Fig 6 (solid brown line) the direct contribution of $p \gamma$ interactions is negligible due to the dominance of the proton synchrotron peak. That is also why Bethe-Heitler pair-production with a lower threshold than photohadronic processes can be neglected against the proton synchrotron emission in magnetic fields of $\mathscr{O}(10 \mathrm{G})$ required to confine the protons within the emitting region of a typical blazar. From Fig. 6 it is also clear that the maxima of the first generation radiation of $e^{ \pm}$and $\gamma$ s from $p \gamma$-interactions are above $10^{28} \mathrm{~Hz}$.

\section{References}

[1] A. A. Abdo et al., Insights into the High-energy $\{\gamma\}$-ray Emission of Markarian 501 from Extensive Multifrequency Observations in the Fermi Era, .

[2] A. A. Abdo et al., Fermi Large Area Telescope Observations Of Markarian 421, .

[3] J. Albert et al., Variable VHE $\gamma$-ray emission from Markarian 501, $0702008 \mathrm{v} 3$.

[4] A. Pichel vol. 8 of International Cosmic Ray Conference, p. 171, 2011. arXiv:1110. 2549.

[5] F. Aharonian et al., An Exceptional Very High Energy Gamma-Ray Flare of PKS 2155-304, .

[6] F. Spanier and M. Weidinger, Hadronic Modeling of AGN Variability, Int. J. Mod. Phys. Conf. Ser. 8 (2012) 293-298.

[7] S. Richter and F. Spanier, A spatially resolved SSC Shock-In-Jet Model, Int. J. Mod. Phys. Conf. Ser. 8 (2012) 392-395, [arXiv:1207.3960].

[8] M. Weidinger, M. Rüger, and F. Spanier, Modelling the steady state spectral energy distribution of the BL-Lac Object PKS 2155-30.4 using a selfconsistent SSC model, Trans. 6 (Jan., 2010) 1-7.

[9] A. Mücke, J. P. Rachen, R. Engel, R. J. Protheroe, and T. Stanev, Photomeson production in astrophysical sources, Nucl. Phys. B Proc. Suppl. 80 (Jan., 2000) C810.

[10] S. R. Kelner and F. A. Aharonian, Energy spectra of gamma rays, electrons, and neutrinos produced at interactions of relativistic protons with low energy radiation, a $\mathrm{X} \mathrm{iv}: 0803.0688$.

[11] M. Boettcher and R. Schlickeiser, The pair production spectrum from photon-photon annihilation., .

[12] R. D. Blandford and A. Konigl, Relativistic jets as compact radio sources, .

[13] S. Richter and F. Spanier, Multi-band implications of external-IC flares, Astroparticle Physics 61 (Feb., 2015) 102-107, [arXiv: 1404 .6943].

[14] R. Reinthal et al., Multi-wavelength Observations of the HBL 1ES 1011+496 in Spring 2008, J. Phys. Conf. Ser. 355 (Mar., 2012) 012017, [arXiv: 1109.6504 ]. 